\title{
Study of Plant Growth Regulators and Micro-Nutrients Response under the Climate Change Scenario in Tomato (Solanum lycopersicum L.)
}

\author{
Pragya Ramgiry* and D. P. Sharma \\ Department of Horticulture, Jawaharlal Nehru Krishi Vishwa Vidyalaya, \\ Jabalpur, M.P., India \\ *Corresponding author
}

\begin{abstract}
A B S T R A C T
Keywords

Tomato, NAA, Boron, Zinc Salicylic Acid and Date of transplanting

Article Info

Accepted: 15 September 2020 Available Online: 10 October 2020

To evaluate the effect of micronutrients and plant growth regulators on different dates of transplanting in tomato, the field experiment was conducted during the Rabi season 2018-19 at the Horticulture Research Complex, Maharajpur, Department of Horticulture. J.N.K.V.V., Jabalpur (M.P.). Significant differences was found for plant height, days to $50 \%$ flowering, days to first picking, TSS, ascorbic acid, lycopene, fruit yield per plot and fruit yield per ha. The highest plant height was recorded at $\mathrm{T}_{25}$. The treatment $\mathrm{T}_{44}$ was found better for days to $50 \%$ flowering and days to first picking. The highest TSS and ascorbic acid was recorded in treatment $\mathrm{T}_{28}$ and $\mathrm{T}_{45}$. The maximum lycopene value was recorded in $\mathrm{T}_{30}$. Significance differences were recorded for the fruit yield per plot and fruit yield per ha in treatment $\mathrm{T}_{13}$.
\end{abstract}

\section{Introduction}

Tomato is one of the valuable crop under the solanaceae family which produces fruits annually. It is native to Western South America and use as a nutritious edible fruit throughout the world that is grown in both greenhouse and field conditions (Smith, 1994). It is an excellent source of many nutrients and secondary metabolities that are important for human health. In tomato lycopene is an important antioxidant which prevents the different forms of cancers such as prostate and lung cancer with the higher nutritive value it is grown all over the world. In India tomato is largely grown in Andhra Pradesh, Madhya Pradesh and Karnataka but mean yield of tomato in India is not according to the crop potential because climate is constantly changing by the human and natural activities. Tomato is comes under the day neutral plant (A day neutral plants is a plant in which flowers regardless of the amount of daylight it receives). Tomato is a warm climate crop $15^{\circ}$ to $30^{\circ} \mathrm{C}$ is best temperature range for its better growth and development. For lycopene development $21^{0}$ to $24^{0} \mathrm{C}$ temperature is required above $35^{\circ} \mathrm{C}$ is not 
congenial for its production. Fluctuation of temperature and climate affect the development of crop in grower field.

Plant growth regulators are used extensively in crop production to improve plant growth and yield by increasing fruit set, fruit number and weight (Batlang, 2008). Salicylic acids play important roles in many physiological process like- enhanced plant growth attributes viz., plant height, number of branches, number of leaves, shoot and root length and total dry biomass of plant at different growth stages and it is stimulant or transmitter of the cell to protect from various climatic factors such as dryness, coldness, heat and also enhanced capacity of plant to withstand several types of stress. Naphthalene acetic acid (NAA) comes under the synthetic plant hormones which regulates the growth and development and also affect the biochemical and physiological process of plant. Helps to promote plant growth by enhancing the cell division, cell elongation and cell differentiation which may initiate the development of plant organs. Flower cluster and whole plant spray of salicylic acid and NAA before the flowering, are highly beneficial.

Micronutrients management is essential to boost the crop production and also increased the fruits quality. Boron and zinc important micronutrient for quality tomato fruit production. Zinc regulates growth and also promotes balanced sugar consumption and Boron helps to providing some nutrients and essential for proper development of their fruits and seeds. These micronutrients are more effective when they are applied as foliar spray. In tomato production different transplanting dates significantly influence quality, growth and fruit yield because different temperature range effect morphologically and physiologically activities of plant. So it is necessities to know which range of temperature and dates of transplanting is suitable for increasing the production of tomato crop.

Production and yield is depending upon the micro climate around the crop. So, for knowing the effect of climatic condition around the crop during production period is a main aspect in modern agriculture production system and there is need to develop a strategies to mitigate the climate affect and response of plant growth regulators and micronutrients for achieving higher fruit yield.

\section{Materials and Methods}

The field experiment was conducted at the Horticulture Research Complex, Maharajpur, Department of Horticulture. J.N.K.V.V., Jabalpur (M.P.) during Rabi season of 20182019. The field experiment was laid out in Randomized Complete Block Design (RCBD) with three replications. Treatment details of the experiment: $\mathrm{T}_{1}$ : Control, $\mathrm{T}_{2}$ : Salicylic acid (1.0mM), $\mathrm{T}_{3:}$ Salicylic acid $(1.5 \mathrm{mM}), \mathrm{T}_{4}$ NAA (25ppm), $\mathrm{T}_{5}$ : NAA (50ppm), $\mathrm{T}_{6}$ : Boron (100ppm), T 7 : Zinc(100ppm), $\mathrm{T}_{8}$ : Salicylic acid $(1.0 \mathrm{mM})+$ Boron (100ppm), $\mathrm{T}_{9}$ : Salicylic acid $(1.5 \mathrm{mM})+$ Boron (100ppm), $\mathrm{T}_{10}$ : Salicylic acid $(1.0 \mathrm{mM})+$ Zinc (100ppm), $\mathrm{T}_{11}$ : Salicylic acid $(1.5 \mathrm{mM})+$ Zinc (100ppm), T12: NAA (25ppm) + Boron (100ppm), T13: NAA (50ppm) + Boron (100ppm), T 14 : NAA (25ppm) + Zinc (100ppm), T15: NAA (50ppm) + Zinc (100ppm). These treatments applied at three different dates of transplanting at 15 days of interval $\left(30^{\text {th }}\right.$ Nov. $), \mathrm{D}_{2}\left(15^{\text {th }}\right.$ Dec.) and $\mathrm{D}_{3}\left(30^{\text {th }}\right.$ Dec.). The seedlings were transplanted at $60 \mathrm{~cm} \times 50 \mathrm{~cm}$.

The details observations recorded on different growth parameters namely plant height at 90 days, days to $50 \%$ flowering, days to first picking, TSS (brix), ascorbic acid (mg/100gm), lycopene $(\mathrm{mg} / 100 \mathrm{gm})$, fruit yield per plot $(\mathrm{kg})$ and fruit yield per ha $(\mathrm{q} / \mathrm{ha})$.The analysis was 
done as per procedure given by Panse and Sukhatme (1967).

\section{Results and Discussion}

The results of the present investigation are presented in Table: 1. The analysis of variance showed the significant differences for all the characters studied (Fig. 1).

The maximum plant height $(152.17 \mathrm{~cm})$ was recorded in the treatment $T_{25}\left(D_{2}+\right.$ Salicylic acid $(1.0 \mathrm{mM})+$ Zinc $(100 \mathrm{ppm}))$ followed by $\mathrm{T}_{15}(148.37 \mathrm{~cm})$ and $\mathrm{T}_{30}(143.23 \mathrm{~cm})$ as compare to other treatments. Similar result found by Ahmed Abou El-Yazied (2011) suggested that foliar spraying with salicylic acid at $100 \mathrm{ppm}$ and chelated zinc at $50 \mathrm{ppm}$ can be used to increase the final yield and fruit quality of sweet pepper plant during the low temperatures of autumn plantations and Choudhary et al., (2016). Among all the treatments, the minimum plant height 114.34 $\mathrm{cm}$ was noticed in the $\mathrm{T}_{4}$.

The significant days to minimum $50 \%$ flowering (58.67 days) was observed in $\mathrm{T}_{44}$ $\left(\mathrm{D}_{3}+\mathrm{NAA}(25 \mathrm{ppm})+\right.$ Zinc $(100 \mathrm{ppm})$ followed by $\mathrm{T}_{43}$ (59 days) and $\mathrm{T}_{42}$ (59.67 days). Similarly days to first picking (67.67 days) recorded earliest in $\mathrm{T}_{44}\left(\mathrm{D}_{3}+\mathrm{NAA}(25\right.$ ppm) + Zinc $(100 \mathrm{pm})$ followed by $\mathrm{T}_{45}(69$ days) and $\mathrm{T}_{43}$ (69.33 days). The significant maximum TSS value (5.22) was found in $\mathrm{T}_{28}$ $\left(\mathrm{D}_{2}+\mathrm{NAA}(50 \mathrm{ppm})+\right.$ Boron $(100 \mathrm{ppm})$ followed by $\mathrm{T}_{43}(5.12)$ and $\mathrm{T}_{30}$ (4.93) and lower value was found in $\mathrm{T}_{37}$ (3.66). Gupta et al., (2018) observed that when crop spray with (NAA@100 ppm + Boron@ 97 ppm) gave the highest TSS value in tomato. The high ascorbic acid (30.42) was noticed in $\mathrm{T}_{45}$ $\left(\mathrm{D}_{3}+\mathrm{NAA}(50 \mathrm{ppm})+\right.$ Zinc $(100 \mathrm{ppm})$ followed by $\mathrm{T}_{29}(29.68)$ and $\mathrm{T}_{42}(29.45)$, while lower value was found in $\mathrm{T}_{16}$ (21.60) (Gupta et al., (2018). Similarly highest lycopene (6.00) was recorded in $\mathrm{T}_{30}\left(\mathrm{D}_{2}+\mathrm{NAA}(50\right.$ $\mathrm{ppm})+$ Zinc $(100 \mathrm{pm})$ followed by $\mathrm{T}_{15}(5.97)$ and $\mathrm{T}_{11}(5.90)$, while lower value was found in $\mathrm{T}_{31}$ (3.10) Pargi et al., (2014). The result showed that the highest fruit yield per plot $(27.447 \mathrm{~kg})$ was noticed in $\mathrm{T}_{13}\left(\mathrm{D}_{1}+\mathrm{NAA}(50\right.$ $\mathrm{ppm})+$ Boron $(100 \mathrm{pm})$ followed by treatment $\mathrm{T}_{30}(27.163 \mathrm{~kg})$ and $\mathrm{T}_{28}(26.920 \mathrm{~kg})$ while the lowest value was found in $T_{31}$ (11.730) (Ali et al., 2015).

Fig.1 Presentation of highest significant value of yield contributing tarits

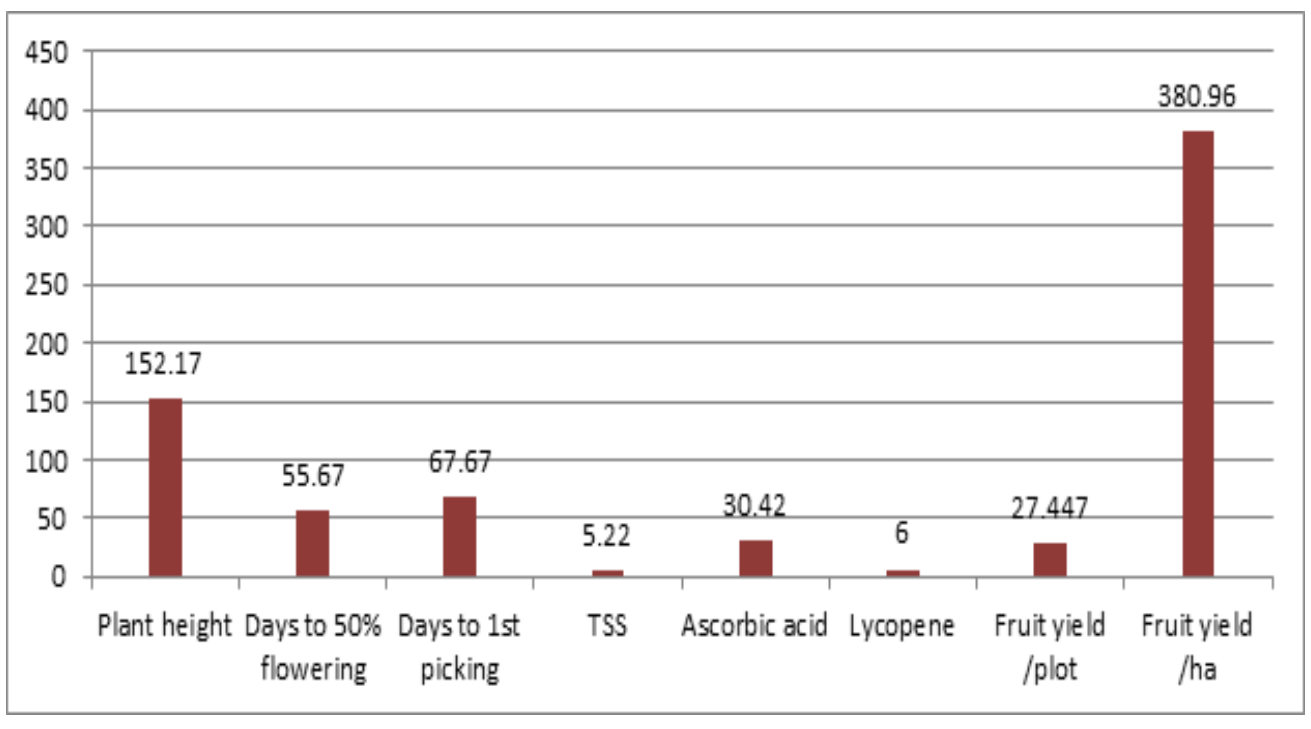


Table.1 Effect of plant growth regulators, micro-nutrients and date of transplanting on tomato

\begin{tabular}{|c|c|c|c|c|c|c|c|c|c|}
\hline Sym. & Treatment details & 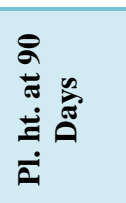 & 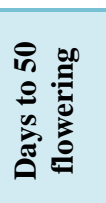 & 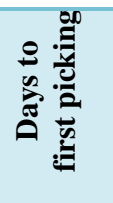 & 象 & 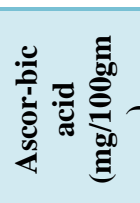 & 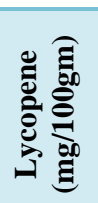 & 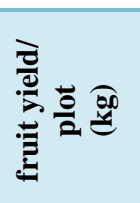 & 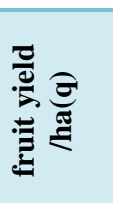 \\
\hline$T_{1}$ & $\mathrm{D}_{1}+$ Control & 116.97 & 68.67 & 84.67 & 3.94 & 21.61 & 3.87 & 17.093 & 237.26 \\
\hline $\mathbf{T}_{2}$ & $\mathrm{D}_{1}+$ Salicylic acid $(1.0 \mathrm{mM})$ & 121.71 & 68.33 & 81.00 & 4.22 & 25.07 & 5.33 & 18.040 & 250.40 \\
\hline $\mathbf{T}_{3}$ & $\mathrm{D}_{1}+$ Salicylic acid $(1.5 \mathrm{mM})$ & 123.12 & 68.67 & 80.00 & 4.32 & 25.90 & 5.47 & 18.930 & 262.75 \\
\hline $\mathbf{T}_{4}$ & $\mathrm{D}_{1}+\mathrm{NAA}(25 \mathrm{ppm})$ & 114.34 & 66.00 & 79.67 & 3.86 & 27.67 & 5.33 & 19.550 & 271.36 \\
\hline$T_{5}$ & $\mathrm{D}_{1}+\mathrm{NAA}(50 \mathrm{ppm})$ & 121.29 & 67.00 & 80.33 & 4.62 & 26.71 & 5.23 & 21.300 & 295.64 \\
\hline$T_{6}$ & $\mathrm{D}_{1}+$ Boron $(100 \mathrm{ppm})$ & 125.52 & 70.00 & 80.67 & 3.96 & 26.90 & 4.07 & 21.557 & 299.21 \\
\hline $\mathbf{T}_{7}$ & $\mathrm{D}_{1}+$ Zinc $(100 \mathrm{ppm})$ & 122.51 & 70.33 & 81.33 & 3.78 & 26.32 & 3.70 & 20.597 & 285.88 \\
\hline $\mathbf{T}_{8}$ & $\mathrm{D}_{1}+$ Salicylic acid $(1.0 \mathrm{mM})+$ Boron $(100 \mathrm{ppm})$ & 124.86 & 67.33 & 81.00 & 4.46 & 26.00 & 5.63 & 21.970 & 304.94 \\
\hline$T_{9}$ & $\mathrm{D}_{1}+$ Salicylicacid $(1.5 \mathrm{mM})+$ Boron $(100 \mathrm{ppm})$ & 130.35 & 68.00 & 79.00 & 4.38 & 25.19 & 5.80 & 23.930 & 332.15 \\
\hline $\mathbf{T}_{10}$ & $\mathrm{D}_{1}+$ Salicylic acid $(1.0 \mathrm{mM})+$ Zinc (100 ppm) & 135.03 & 70.67 & 81.67 & 4.01 & 24.56 & 5.67 & 23.067 & 320.16 \\
\hline $\mathbf{T}_{11}$ & $\mathrm{D}_{1}+$ Salicylicacid $(1.5 \mathrm{mM})+$ Zinc $(100 \mathrm{ppm})$ & 129.29 & 68.33 & 81.33 & 4.30 & 25.77 & 5.90 & 25.893 & 359.40 \\
\hline $\mathbf{T}_{12}$ & $\mathrm{D}_{1}+\mathrm{NAA}(25 \mathrm{ppm})+$ Boron $(100 \mathrm{ppm})$ & 134.17 & 70.00 & 81.00 & 4.33 & 27.09 & 5.10 & 26.373 & 366.06 \\
\hline$T_{13}$ & $\mathrm{D}_{1}+\mathrm{NAA}(50 \mathrm{ppm})+$ Boron $(100 \mathrm{ppm})$ & 130.72 & 66.67 & 79.00 & 4.49 & 27.86 & 5.30 & 27.447 & 380.96 \\
\hline $\mathbf{T}_{14}$ & $\mathrm{D}_{1}+\mathrm{NAA}(25 \mathrm{ppm})+$ Zinc $(100 \mathrm{ppm})$ & 134.39 & 68.67 & 80.00 & 3.87 & 28.79 & 5.10 & 23.440 & 325.35 \\
\hline$T_{15}$ & $\mathrm{D}_{1}+\mathrm{NAA}(50 \mathrm{ppm})+\operatorname{Zinc}(100 \mathrm{ppm})$ & 148.37 & 68.00 & 79.00 & 4.03 & 29.35 & 5.97 & 26.490 & 367.68 \\
\hline$T_{16}$ & $\mathrm{D}_{2}+$ Control & 119.30 & 71.33 & 81.00 & 3.86 & 21.60 & 4.03 & 17.273 & 239.75 \\
\hline $\mathbf{T}_{17}$ & $\mathrm{D}_{2}+$ Salicylic acid $(1.0 \mathrm{mM})$ & 122.03 & 68.33 & 79.67 & 4.04 & 25.03 & 5.40 & 18.403 & 255.44 \\
\hline$T_{18}$ & $\mathrm{D}_{2}+$ Salicylic acid $(1.5 \mathrm{mM})$ & 125.53 & 68.00 & 80.33 & 4.30 & 23.38 & 5.33 & 18.660 & 259.00 \\
\hline$T_{19}$ & $\mathrm{D}_{2}+\mathrm{NAA}(25 \mathrm{ppm})$ & 120.83 & 68.00 & 79.00 & 3.89 & 26.98 & 5.50 & 19.023 & 264.04 \\
\hline $\mathbf{T}_{20}$ & $\mathrm{D}_{2}+\mathrm{NAA}(50 \mathrm{ppm})$ & 121.93 & 67.33 & 78.00 & 4.24 & 26.06 & 5.23 & 21.443 & 297.63 \\
\hline $\mathbf{T}_{21}$ & $\mathrm{D}_{2}+$ Boron $(100 \mathrm{ppm})$ & 126.20 & 68.00 & 78.33 & 3.71 & 26.96 & 5.10 & 20.557 & 285.33 \\
\hline$T_{22}$ & $\mathrm{D}_{2}+\operatorname{Zinc}(100 \mathrm{ppm})$ & 126.17 & 67.67 & 79.33 & 3.67 & 27.17 & 5.13 & 21.030 & 291.90 \\
\hline $\mathbf{T}_{23}$ & $\mathrm{D}_{2}+$ Salicylic acid $(1.0 \mathrm{mM})+$ Boron $(100 \mathrm{ppm})$ & 127.27 & 67.67 & 80.00 & 4.48 & 25.44 & 5.60 & 21.270 & 295.23 \\
\hline $\mathbf{T}_{24}$ & $\mathrm{D}_{2}+$ Salicylicacid $(1.5 \mathrm{mM})+$ Boron $(100 \mathrm{ppm})$ & 131.23 & 66.33 & 78.00 & 4.65 & 25.46 & 5.87 & 24.123 & 334.83 \\
\hline $\mathbf{T}_{25}$ & $\mathrm{D}_{2}+$ Salicylic acid $(1.0 \mathrm{mM})+$ Zinc $(100 \mathrm{ppm})$ & 152.17 & 69.67 & 78.00 & 4.10 & 25.92 & 5.70 & 23.283 & 323.17 \\
\hline $\mathbf{T}_{26}$ & $\mathrm{D}_{2}+$ Salicylicacid $(1.5 \mathrm{mM})+$ Zinc $(100 \mathrm{ppm})$ & 130.17 & 69.33 & 76.33 & 4.07 & 26.38 & 5.70 & 25.940 & 360.05 \\
\hline $\mathbf{T}_{27}$ & $\mathrm{D}_{2}+\mathrm{NAA}(25 \mathrm{ppm})+$ Boron $(100 \mathrm{ppm})$ & 130.83 & 68.67 & 80.00 & 4.47 & 28.07 & 5.50 & 26.853 & 372.72 \\
\hline$T_{28}$ & $\mathrm{D}_{2}+$ NAA $(50 \mathrm{ppm})+$ Boron $(100 \mathrm{ppm})$ & 133.03 & 69.00 & 76.00 & 5.22 & 29.01 & 5.73 & 26.920 & 373.65 \\
\hline $\mathbf{T}_{29}$ & $\mathrm{D}_{2}+\mathrm{NAA}(25 \mathrm{ppm})+\mathrm{Zinc}(100 \mathrm{ppm})$ & 136.63 & 68.67 & 79.00 & 4.44 & 29.68 & 5.40 & 24.040 & 333.67 \\
\hline $\mathbf{T}_{30}$ & $\mathrm{D}_{2}+\mathrm{NAA}(50 \mathrm{ppm})+$ Zinc $(100 \mathrm{ppm})$ & 143.23 & 69.33 & 76.67 & 4.93 & 29.35 & 6.00 & 27.163 & 377.03 \\
\hline $\mathbf{T}_{31}$ & $\mathrm{D}_{3}+$ Control & 116.57 & 66.00 & 74.67 & 4.12 & 22.33 & 3.10 & 11.730 & 162.81 \\
\hline $\mathbf{T}_{32}$ & $\mathrm{D}_{3}+$ Salicylic acid $(1.0 \mathrm{mM})$ & 121.90 & 65.33 & 72.33 & 4.23 & 26.23 & 4.40 & 14.223 & 197.42 \\
\hline $\mathbf{T}_{33}$ & $\mathrm{D}_{3}+$ Salicylic acid $(1.5 \mathrm{mM})$ & 121.83 & 64.33 & 71.67 & 4.44 & 26.45 & 4.73 & 12.860 & 178.50 \\
\hline $\mathbf{T}_{34}$ & $\mathrm{D}_{3}+\mathrm{NAA}(25 \mathrm{ppm})$ & 117.83 & 63.33 & 75.67 & 4.00 & 27.02 & 4.77 & 13.530 & 187.80 \\
\hline $\mathbf{T}_{35}$ & $\mathrm{D}_{3}+\mathrm{NAA}(50 \mathrm{ppm})$ & 122.53 & 63.00 & 72.33 & 4.89 & 27.48 & 4.60 & 14.603 & 202.69 \\
\hline $\mathbf{T}_{36}$ & $\mathrm{D}_{3}+$ Boron $(100 \mathrm{ppm})$ & 123.00 & 62.33 & 73.00 & 3.94 & 27.24 & 4.47 & 16.227 & 225.23 \\
\hline $\mathbf{T}_{37}$ & $\mathrm{D}_{3}+\operatorname{Zinc}(100 \mathrm{ppm})$ & 123.83 & 62.00 & 75.00 & 3.66 & 27.09 & 4.20 & 13.890 & 192.79 \\
\hline $\mathbf{T}_{38}$ & $\mathrm{D}_{3}+$ Salicylic acid $(1.0 \mathrm{mM})+$ Boron $(100 \mathrm{ppm})$ & 123.93 & 60.33 & 72.67 & 4.79 & 23.65 & 4.60 & 16.540 & 229.58 \\
\hline$T_{39}$ & $\mathrm{D}_{3}+$ Salicylicacid $(1.5 \mathrm{mM})+$ Boron $(100 \mathrm{ppm})$ & 125.63 & 61.33 & 72.00 & 4.77 & 26.35 & 4.93 & 19.247 & 267.15 \\
\hline $\mathbf{T}_{40}$ & $\mathrm{D}_{3}+$ Salicylic acid $(1.0 \mathrm{mM})+$ Zinc (100 ppm) & 141.37 & 60.67 & 71.33 & 4.35 & 25.26 & 4.90 & 17.127 & 237.72 \\
\hline $\mathbf{T}_{41}$ & $\mathrm{D}_{3}+$ Salicylicacid $(1.5 \mathrm{mM})+$ Zinc $(100 \mathrm{ppm})$ & 126.13 & 61.33 & 71.00 & 4.54 & 26.19 & 4.87 & 21.857 & 303.37 \\
\hline $\mathbf{T}_{42}$ & $\mathrm{D}_{3}+\mathrm{NAA}(25 \mathrm{ppm})+$ Boron $(100 \mathrm{ppm})$ & 134.63 & 59.67 & 71.67 & 4.86 & 29.45 & 4.87 & 19.320 & 268.16 \\
\hline $\mathbf{T}_{43}$ & $\mathrm{D}_{3}+\mathrm{NAA}(50 \mathrm{ppm})+$ Boron $(100 \mathrm{ppm})$ & 127.17 & 59.00 & 69.33 & 5.12 & 29.38 & 4.73 & 22.290 & 309.38 \\
\hline $\mathbf{T}_{44}$ & $\mathrm{D}_{3}+\mathrm{NAA}(25 \mathrm{ppm})+$ Zinc $(100 \mathrm{ppm})$ & 131.07 & 58.67 & 67.67 & 4.29 & 27.09 & 4.87 & 17.497 & 242.86 \\
\hline \multirow[t]{3}{*}{$\mathbf{T}_{45}$} & $\mathrm{D}_{3}+\mathrm{NAA}(50 \mathrm{ppm})+\mathrm{Zinc}(100 \mathrm{ppm})$ & 139.50 & 60.00 & 69.00 & 4.80 & 30.42 & 5.27 & 20.107 & 279.08 \\
\hline & $\operatorname{SEm} \pm$ & 3.23 & 1.57 & 1.46 & 0.13 & 0.72 & 0.26 & 1.23 & 17.19 \\
\hline & C.D. at $5 \%$ level & 9.13 & 4.46 & 4.15 & 0.38 & 2.04 & 0.75 & 3.50 & 48.64 \\
\hline
\end{tabular}


The maximum fruit yield per ha (380.96 q/ha) was noticed in treatment $\mathrm{T}_{13}\left(\mathrm{D}_{1}+\mathrm{NAA}(50\right.$ $\mathrm{ppm})+$ Boron (100pm) followed by $\mathrm{T}_{30}$ (377.03 q/ha) and $\mathrm{T}_{28}(373.65 \mathrm{q} / \mathrm{ha})$, while lower was found in $\mathrm{T}_{13}(162.81 \mathrm{q} / \mathrm{ha})$. Londhe, Mahadev Bapu (2018) noticed that the maximum yield (360.82 q/ha) was recorded in treatment where NAA + Boric acid $0.2 \%$ was applied.

\section{References}

Abou El-Yazied, A. (2011). Effect of Foliar Application of Salicylic Acid and Chelated Zinc on Growth and Productivity of Sweet Pepper (Capsicum annuum L.) under Autumn Planting. Research Journal of Agriculture and Biological Sciences, 7(6): 423-433.

Ali, M. R., Mehraj, H. and Jamal Uddin, AFM. (2015). Effects of foliar application of zinc and boron on and yield of summer tomato. Journal of Bioscience and Agriculture Research, 6(1): 512-517.

Batlang, U. (2008). Benzylvadenine plus gibberellins (GA4+7) increase fruit size and yield in greenhouse-grown hot pepper (Capsicum annuum L.). Journal of Biological Science, 8(3): 659-662.

Choudhary, A., Mishra, A., Bola, P. K., Moond, S. K. and Dhayal, M. (2016).
Effect of foliar application of zinc and salicylic acid on growth, flowering and chemical constitute of African marigold cv. pusa narangi gainda (Targets erecta L.). Journal of Applied and Natural Science, 8 (3): 1467-1470.

Gupta, S., Bisen, R. K., Verma, S. and Sharma, G. (2018). Study on effect of plant growth regulators and boron on quality attributes of tomato (Solanum lycopersicum MILL.). Journal of Pharmacognosy and Phytochemistry, 7(4): 2581-2583.

Londhe, Mahadev Bapu (2018). Effect of plant growth regulators and micronutrients on fruit setting, yield and quality of tomato during summer season. M.Sc. Thesis, MPKV, Rahuri.32p

Panse, V. G. and Sukhatme, P. V. (1967). Statistical methods for Agricultural workers, Publication ICAR, Publication New Delhi, pp.152-161.

Pargi, S. C., Lal, E.P., Singh, N. and Biswas, T.K. (2014).Effect of Naphthalene acetic acid on Biochemical parameters, Growth and Yield of Tomato (Lycopersicon esculentum Mill.). Journal of Agriculture and Veterinary Science, 7(7): 16-18.

Smith, A. f. (1994). The Tomato in America: Early History, Culture, and Cookery. Columbia SC, USA: University of South Carolina Press.

\section{How to cite this article:}

Pragya Ramgiry and Sharma, D. P. 2020. Study of Plant Growth Regulators and MicroNutrients Response under the Climate Change Scenario in Tomato (Solanum lycopersicum L.). Int.J.Curr.Microbiol.App.Sci. 9(10): 1868-1872. doi: https://doi.org/10.20546/ijcmas.2020.910.228 